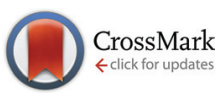

Cite this: Org. Chem. Front., 2014, 1 , 834

Received 24th March 2014, Accepted 12th June 2014

DOI: $10.1039 / c 4 q 000088 a$

rsc.li/frontiers-organic

\section{Modern multicomponent reactions for better drug syntheses}

\author{
Tryfon Zarganes-Tzitzikas and Alexander Dömling*
}

The ever growing structural complexity of modern drugs has led to an increase in the difficulty of synthetic efforts for their production (Fig. 1). This general trend can be rationalized as more complex proteins and enzymes are emerging as viable drug targets and there is an increasing need for higher selectivity and potency of compounds.

The large scale GMP synthesis of complex drugs is almost automatically reflected in the considerably higher cost-ofgoods. Sometimes, however, a cleverly designed synthetic

Department for Drug Design, University of Groningen, A. Deusinglaan 1, $9700 A D$ Groningen, The Netherlands. E-mail: a.s.s.domling@rug.nl;

http://www.drugdesign.nl/ pathway can lead to rapid, convergent, and cheaper syntheses of otherwise difficult and lengthy-to-access drug targets. An interesting recent case is the 2013 FDA approved hepatitis C NS3 protease inhibitor Telaprevir.

Hepatitis $\mathrm{C}$ is a viral infectious disease affecting more than 200 million people worldwide and is currently treated by a combination of PEGylated interferon and ribavirin. However, a significant number of patients do not respond to this therapy due to adverse effects or viral rebound caused by resistant strains. The recent approval of two peptidic HCV NS3 protease inhibitors Telaprevir and Boceprevir has shifted the HCV treatment paradigm and gives patients new and powerful treatment options. The reported technical synthesis of Telaprevir involves

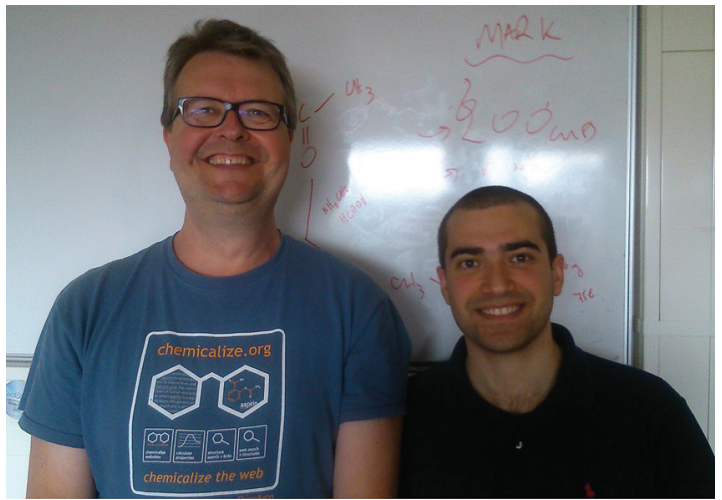

Zarganes-Tzitzikas Tryfon and Alexander Dömling
Zarganes-Tzitzikas Tryfon was born in Thessaloniki, Greece in 1988. He obtained his B.Sc. degree in Chemistry from the Aristotle University of Thessaloniki in 2010. In March 2012 he received his M.Sc. degree with emphasis on Organic Chemistry under the guidance of Professors J. Stephanidou-Stephanatou and $K$. Tsoleridis from the Aristotle University of Thessaloniki. Since July 2012 he has been pursuing his Ph.D. in Medicinal Chemistry under the guidance of Prof. Alexander Dömling in the Drug Design Group at the University of Gröningen, The Netherlands.

Alexander Dömling has been holding the chair for Drug Design at the University of Groningen since 2011. He studied Chemistry \& Biology at the Technische Universität München and obtained his Ph.D. under the guidance of Ivar Ugi. After a postdoc under a Humboldt fellowship in the group of the Nobel Laureate Barry Sharpless he founded the biotech company Morphochem and later Carmolex Inc. After his habilitation he worked as full professor at the University of Pittsburgh in the School of Pharmacy. His interests are centered around multicomponent reaction chemistry (MCR) and its application to problems in drug design. His special focus is on MCR centered pharmacophore methods, structure-baseddrug-design and MCR centered fragment-based-drug-design. He is the author of more than 150 scientific articles, reviews and book contributions. He has applied for more than 30 patents. His long term vision is to bring a novel drug to patients in an indicated area of unmet medical needs. 


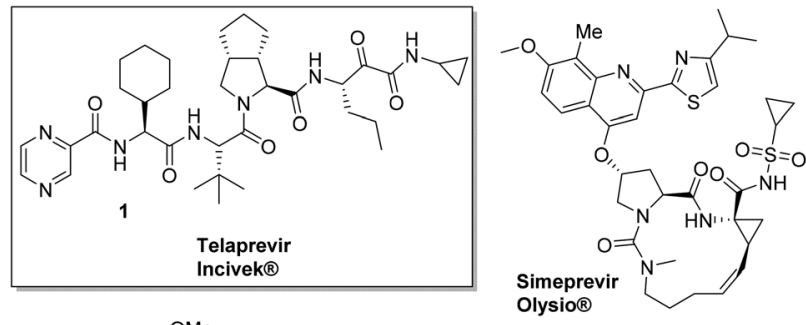<smiles>COc1cnc(O)c2cc(Cl)ccc12</smiles>
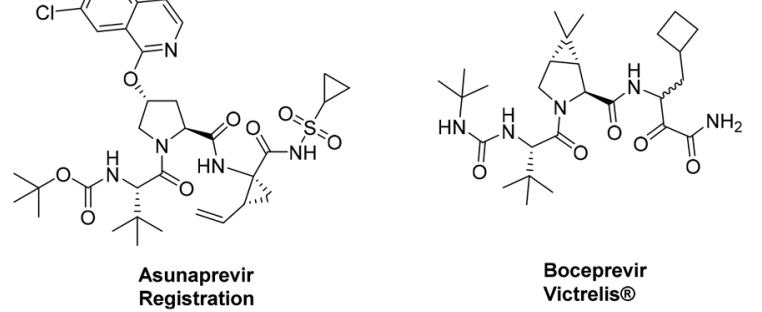

Fig. 1 Structural complexity of marketed or late-stage HCV NS3 inhibitors.

a lengthy, highly linear strategy relying on standard peptide chemistry and entails more than 20 linear steps. ${ }^{1}$ For example, the central bicyclic proline derivative is synthesized in racemic form via a nine-step sequence. The desired enantiomer is only obtained after chiral HPLC separation. Optimization of the synthesis of Telaprevir could significantly lower the production costs, thereby making this highly needed drug available to an increased proportion of the world population in the future.

Here we highlight the work of two independent groups towards significant improvements in the synthesis of the HCV drug Telaprevir 1. It has to be noted that both syntheses are currently being investigated for large scale preparation by a pharmaceutical company. Recently Orru and Ruijter et al. from the VU University Amsterdam disclosed a highly efficient and stereoselective synthesis of Telaprevir (Incivek®) employing the combined use of biocatalysts and multicomponent reactions (MCRs). The synthesis comprises of only eleven steps in total compared to twenty-four in the originally reported procedure. ${ }^{2-4}$ The significance of the work is a reduction by more than half the steps while comprising a "greener" process which can be additionally translated into a considerably lower cost-of-goods.

A retrosynthetic analysis of $\mathbf{1}$ involving two MCRs is presented in Fig. 2. The required starting materials for the key Ugi-type 3CR were the carboxylic acid 2, cyclic imine 3, and isocyanide $\mathbf{4}$. The acid 2 could be accessed by standard peptide chemistry, while the chiral imine 3 was generated in situ from commercially available 5 by the enzyme MAO-N catalyzed oxidation. The isocyanide $\mathbf{4}$ was accessed via a Passerini threecomponent reaction (P-3CR) of compounds 6 and 7, and acetic acid.

Coupling of pyrazinecarboxylic acid 9 and L-cyclohexylglycine methyl ester $\mathbf{1 0}$ followed by saponification afforded $\mathbf{1 1}$ in excellent yield. Subsequent coupling with $\mathrm{L}$-tert-leucine methyl ester $\mathbf{1 2}$ and saponification furnished the required optically

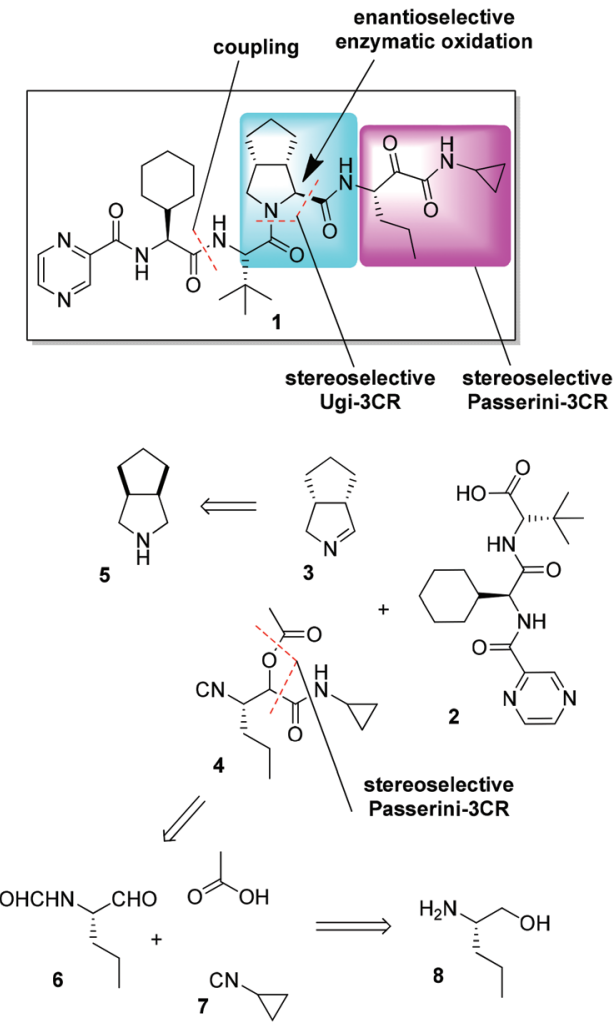

Fig. 2 MCR retrosynthetic analysis of Telaprevir (Incivek®).

pure acid 2. The Orru and Ruijter group was able to significantly increase both the atom and step economy and the overall yield ( $74 \%$ vs. $11 \%$ over four steps) of the acid 2 compared to the already known synthesis ${ }^{1}$ (Fig. 3).

The construction of the isocyanide fragment $\mathbf{4}$ was done in three steps in which a Passerini 3-component reaction (P-3CR) played a key role. Commercial (S)-2-amino-1-pentanol 8 was transformed to formamide $\mathbf{1 3}$ by $\mathrm{N}$-formylation. Following the work of Ngouansavanh and $\mathrm{Zhu},{ }^{5}$ the in situ oxidation of alcohol 13 and a P-3CR was performed in one pot giving access to 14. Both the alcohol oxidation and the Passerini reac-

$$
\text { (1) }
$$

Fig. 3 Synthesis of acid 2. Reagents and conditions: (a) BOP, $\mathrm{Et}_{3} \mathrm{~N}, \mathrm{DMF}$, 98\%; (b) $\mathrm{NaOH}, \mathrm{THF}-\mathrm{H}_{2} \mathrm{O}-\mathrm{MeOH}, 95 \%$; (c) H-Tle-OMe 12, EDC, HOAt, DMF, 84\%; (d) $\mathrm{NaOH}, \mathrm{THF}-\mathrm{H}_{2} \mathrm{O}-\mathrm{MeOH}, 95 \%$. BOP = benzotriazole-1yl-oxy-tris-(dimethylamino)-phosphonium hexafluorophosphate, $\mathrm{DMF}=$ dimethylformamide, EDC = 1-ethyl-3-(3 dimethyl-aminopropyl)carbodiimide, HOAt = 1-hydroxy-7-azabenzotriazole. 


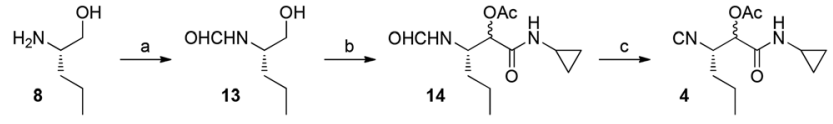

Fig. 4 Synthesis of isocyanide 4. Reagents and conditions: (a) EtOCHO, $\Delta$, 99\%; (b) DMP, cyclopropyl isocyanide, $\mathrm{CH}_{2} \mathrm{Cl}_{2}, 60 \%$; (c) triphosgene, $\mathrm{NMM}, \mathrm{CH}_{2} \mathrm{Cl}_{2},-30{ }^{\circ} \mathrm{C}, 87 \%$. DMP $=$ Dess-Martin periodinane, $\mathrm{NMM}=$ $\mathrm{N}$-methylmorpholine.

tion were performed in $\mathrm{CH}_{2} \mathrm{Cl}_{2}$. Thus, one-pot Dess-Martin oxidation/Passerini reaction of $\mathbf{1 3}$ furnished 14 in $60 \%$ yield. Dehydration then afforded the required isocyanide $\mathbf{4}$ in very good yield $(87 \%)$. Importantly, no racemization of the C3 stereocenter in $\mathbf{4}$ was observed. The diastereomeric stereocenter of 4 in $\mathrm{C} 4$ will be removed at a later stage of the synthesis. The crucial building block $\mathbf{4}$ was thus accessible in only three steps from commercially available starting materials (Fig. 4).

The last step towards the three-component Ugi-type coupling envisaged in the retrosynthesis is described below. The commercial amine 5 was oxidized to imine 3 ( $94 \%$ ee) by MAO-N as previously described. ${ }^{6-8}$ Reacting cyclic Schiff base 3 with the acid 2 and the isocyanide 4 gave the advanced intermediate 15. Finally, cleavage of the acetate followed by DessMartin oxidation gave Telaprevir 1 as a $83: 13: 4$ mixture of diastereomers, with one minor diastereomer derived from the incomplete stereoinduction of the Ugi-type 3CR, and the other from the minor enantiomer of imine 3. Chromatography allowed straightforward separation of the diastereomers to afford pure Telaprevir $\mathbf{1}$ in $80 \%$ yield over the last two steps (Fig. 5). The above synthesis comprises of only eleven steps in total (technical 24), with seven steps in the longest linear sequence and a yield of $45 \%$ starting from 10 . In conclusion, the MCR approach that the group of Orru and Ruijter proposes gives access to Telaprevir $\mathbf{1}$ in higher yields and with higher efficiency than any previously disclosed process. Furthermore, the chiral information used for the preparation is derived from readily available and inexpensive building blocks, making the process a highly effective approach to such prolyl dipeptides.

Recently Banfi et al. disclosed an alternative pathway to Telaprevir combining biocatalysis and MCRs. ${ }^{9,10}$ Their strategy involved the construction of the backbone of the C-terminus of the target compounds while subsequently forming the bicyclic pyrrolidine. Biocatalytic desymmetrization of meso diol 16 followed by a short sequence of standard transformations gives alcohol $17 .^{11}$

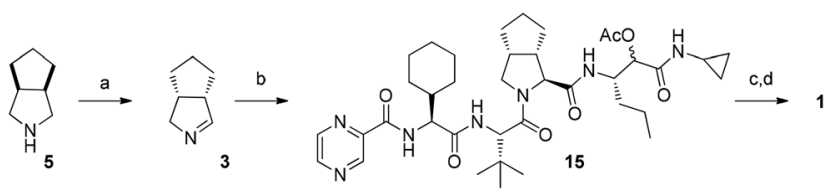

Fig. 5 Synthesis of 1 . Reagents and conditions: (a) monoamine oxidase (MAO-N), $100 \mathrm{mM} \mathrm{KPO} 4$ buffer, $\mathrm{pH}=8.0,37^{\circ} \mathrm{C}$; (b) 2, 4, $\mathrm{CH}_{2} \mathrm{Cl}_{2}, 76 \%$; (c) $\mathrm{K}_{2} \mathrm{CO}_{3}, \mathrm{MeOH}$; (d) DMP, $\mathrm{CH}_{2} \mathrm{Cl}_{2}, 80 \%$ over two steps. DMP = DessMartin periodinane.

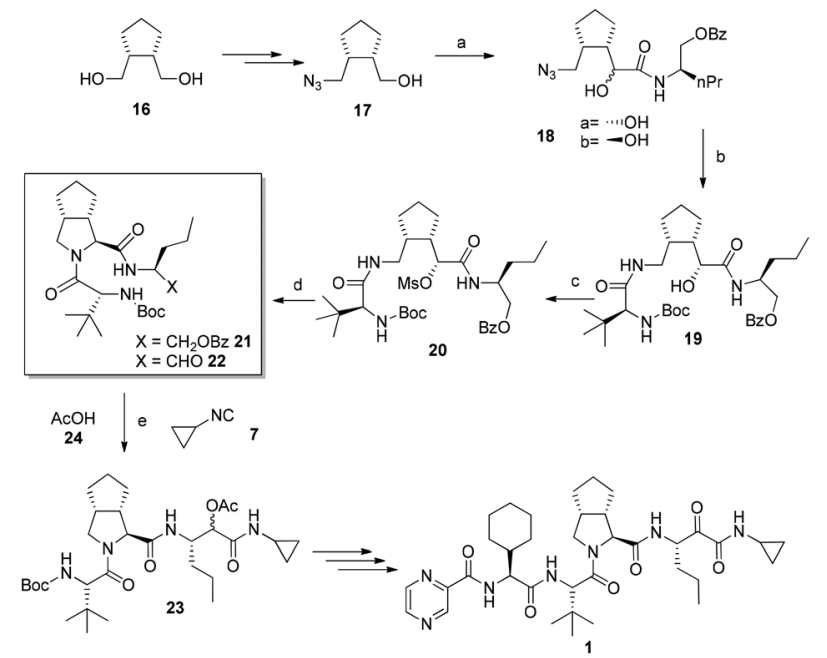

Fig. 6 Synthesis of 1 by Banfi et al. Reagents and conditions: (a) (i) DMSO, oxalyl chloride in DCM, (ii) (S)-2-isocyanopentyl benzoate, boric acid in $\mathrm{CH}_{3} \mathrm{CN}, 92 \%$ diastereoisomeric mixture a/b = $2: 1$; (b) (i) $\mathrm{PPh}_{3}-$ $\mathrm{H}_{2} \mathrm{O}$, (ii) NMM, L-Boc-t-leucine, PyBoP, 78\%; (c) methanesulfonyl chloride, $\mathrm{Et}_{3} \mathrm{~N}$ in DCM; (d) (i) $\mathrm{NaH}, \mathrm{DMF}, 78 \%$; (e) 7, 24 and 22 in DCM, r.t. $\mathrm{DMF}=$ dimethylformamide, $\mathrm{NMM}=\mathrm{N}$-methylmorpholine, $\mathrm{PyBoP}=$ benzotriazol-1-yl-oxytripyrrolidinophosphonium hexafluorophosphate.

Next, in situ oxidation of $\mathbf{1 7}$ to the aldehyde is done in a Passerini-reaction to afford $\mathbf{1 8}$ as a mixture of the diastereoisomer ratio of $\mathbf{a} / \mathbf{b}=2: \mathbf{1}$. After the azide reduction of $\mathbf{1 8 a}$, an intramolecular $\mathrm{O}$ to $\mathrm{N}$ migration occurs to give the amidoalcohol 19. Cyclization via the mesylate 20 proceeded in excellent yields to give 21 (Fig. 6). After deprotection and oxidation, aldehyde 22 is formed. A Passerini reaction takes place in the next step involving compound 22, cyclopropyl isocyanide 24, and acetic acid 25 producing compound 23, which after three steps is converted into Telaprevir. As an additional benefit of the synthesis the volatile cyclopropylisocyanide is not isolated but produced in situ from the formamide precursor. The second route by Banfi et al. although longer than the first one is highlighting alternative synthetic processes which might find use due to intellectual property management and/or better yields and stereoselectivities.

The reduction of the steps of the technical total synthesis of Telaprevir by more than $1 / 2$ using two convergent MCRs in combination with biocatalysis is indeed an intriguing piece of applied chemistry. It should be noted that Telaprevir is an outstandingly complex example of process improvement by MCR; however, an increasing number of drugs have been recently synthesized by this technology. ${ }^{12-17}$

\section{Acknowledgements}

Funding is provided for $\mathrm{AD}$ by the National Institute of Health (1R21M087617-01), European Lead Factory (IMI under grant agreement no. 115489) and the Qatar National Research Foundation (NPRP6-065-3-012). 


\section{Notes and references}

1 Y. Yip, F. Victor, J. Lamar, R. Johnson, Q. M. Wang, J. I. Glass, N. Yumibe, M. Wakulchik, J. Munroe and S. H. Chen, Bioorg. Med. Chem. Lett., 2004, 14, 5007.

2 A. Znabet, M. M. Polak, E. Janssen, F. J. de Kanter, N. J. Turner, R. V. Orru and E. Ruijter, Chem. Commun., 2010, 46, 7918.

3 E. Ruijter, R. Orru, A. Znabet, M. Polak and N. Turner, WO/ 2011/103932, VUA, 2011.

4 E. Ruijter, R. Orru, A. Znabet, M. Polak and N. Turner, WO/ 2011/103933, VUA, 2011.

5 T. Ngouansavanh and J. Zhu, Angew. Chem., Int. Ed., 2006, 45, 3495.

6 V. Köhler, K. R. Bailey, A. Znabet, J. Raftery, M. Helliwell and N. J. Turner, Angew. Chem., Int. Ed., 2010, 49, 2182.

7 B. Mijts, et al., WO/2010/008828, Codexis, Inc., 2010.
8 A. Znabet, et al., Angew. Chem., Int. Ed., 2010, 49, 52895292.

9 L. Banfi, A. Basso, L. Moni and R. Riva, Eur. J. Org. Chem., 2014, 2005-2015.

10 F. Morana, A. Basso, R. Riva, V. Rocca and L. Banfi, Chem. Eur. J., 2013, 19, 4563.

11 R. Riva, et al., WO/2013/178682, Chemo Eberica, S.A., 2013.

12 A. Dömling, W. Wang and K. Wang, Chem. Rev., 2012, 112, 3083.

13 H. P. Cao and A. Dömling, Chem. - Eur. J., 2010, 16, 12296.

14 H. Liu, S. William, E. Herdtweck, S. Botros and A. Dömling, Chem. Biol. Drug Des., 2012, 79, 470.

15 A. D. Borthwick, et al., J. Med. Chem., 2012, 55, 783.

16 J. W. Clader, et al., J. Med. Chem., 1996, 39, 3684.

17 D. A. Burnett, M. A. Caplen, H. R. Davis, R. E. Burrier and J. W. Clader, J. Med. Chem., 1994, 37, 1733. 